\title{
Il gozzo multinodulare
}

\author{
Alessandro Brancatella ${ }^{1}$. Nicola Viola ${ }^{1}$. Daniele Sgrò ${ }^{1}$. Debora Ricci ${ }^{1}$ Paolo Vitti ${ }^{1}$. Ferruccio Santini ${ }^{1}$. \\ Francesco Latrofa ${ }^{1}$
}

Accettato: 27 dicembre 2020 / Pubblicato online: 23 agosto 2021

(c) The Author(s) 2021

\begin{abstract}
Sommario Si definisce gozzo l'incremento diffuso o nodulare della ghiandola tiroidea. Il suo sviluppo è legato a fattori genetici e ambientali, di cui il più importante è rappresentato dalla carenza iodica. L'inquadramento clinico prevede un'attenta valutazione dei sintomi, dei segni, dei risultati degli esami ormonali, delle caratteristiche ecografiche e citologiche. Il trattamento deve essere poi individualizzato tenendo conto della disponibilità di molteplici opzioni terapeutiche.
\end{abstract}

Parole chiave Carenza iodica $\cdot$ Iodio $\cdot$ Autonomia funzionale $\cdot$ Tiroidectomia $\cdot$ Levotiroxina

\section{Introduzione}

Con il termine "gozzo" si definisce una tiroide di dimensioni aumentate in assenza di patologie autoimmuni o neoplastiche. Classicamente il gozzo viene suddiviso in endemico, sporadico o familiare. Nella realtà, queste tre entità non sono distinguibili da un punto di vista clinico ma unicamente per la prevalenza di fattori genetici o ambientali che ne favoriscono lo sviluppo. In base alla sua morfologia, distinguiamo poi un gozzo diffuso senza noduli tipico dell'età giovanile e un gozzo multinodulare più frequentemente riscontrato nell'età avanzata. Nel contesto del gozzo, i noduli possono essere singoli o multipli. Inoltre, è possibile che uno o più no-

Proposto da Francesco Trimarchi.

Informazioni Supplementari La versione online contiene materiale supplementare disponibile su

https://doi.org/10.1007/s40619-021-00926-0.

$\triangle$ F. Latrofa

francesco.latrofa@unipi.it

1 U.O. Endocrinologia 1, Dipartimento di Medicina Clinica e Sperimentale, Università di Pisa, Pisa, Italia duli producano un eccesso di ormoni tiroidei causando ipertiroidismo oppure, nella maggioranza dei casi, che i noduli non siano funzionanti. Con la diffusione di esami di imaging altamente sensibili (in primo l'ecografia), negli ultimi anni è cresciuta sensibilmente il numero di soggetti affetti da noduli tiroidei, raggiungendo quasi il 50\% della popolazione, soprattutto di sesso femminile. La necessità di identificare la piccola percentuale di noduli maligni ha portato allo sviluppo negli ultimi anni di linee guida, consensi di esperti e flow-chart con l'obiettivo di razionalizzare l'utilizzo degli strumenti diagnostici e terapeutici [1].

\section{Carenza iodica e gozzo endemico}

La prevalenza di gozzo diffuso o nodulare è strettamente correlata all'apporto iodico nella popolazione. La carenza iodica rappresenta il principale fattore di rischio per lo sviluppo del gozzo poiché questo elemento è essenziale per la sintesi degli ormoni tiroidei [1]. La maggior parte dello iodio è contenuta nell' acqua degli oceani, mentre la sua disponibilità nell'acqua potabile e nei cibi vegetali e animali è limitata in molte aree del pianeta. Il gozzo endemico è più diffuso nelle zone interne e montuose del pianeta ma si ritrova anche in zone costiere. Solo poche aree geografiche sono iodiosufficienti e in molte regioni del pianeta sono state adottate politiche sanitarie volte all'eradicazione della carenza iodica mediante l'utilizzo di sale iodato e di cibi fortificati con lo iodio. Nell'ultima analisi mondiale sulla carenza iodica del 2020, ancora una percentuale non trascurabile della popolazione mondiale è esposta alla carenza iodica, valutata come escrezione urinaria dello iodio [2]. La più bassa prevalenza di carenza iodica viene registrata nell'America settentrionale, dove oltre il $90 \%$ della popolazione consuma abitualmente sale iodato. È interessante notare come ancora la popolazione di numerosi paesi europei sviluppati sia iodio-carente, 
Tabella 1 Fabbisogno giornaliero di iodio

\begin{tabular}{lc}
\hline Fascia di età & Fabbisogno giornaliero $(\mu \mathrm{g})$ \\
\hline Bambini 0-5 anni & 90 \\
Bambini 6-12 anni & 120 \\
Adulti > 12 anni & 150 \\
Gravidanza & 250 \\
Allattamento & 250 \\
\hline
\end{tabular}

data l'assenza di politiche sanitarie mirate a contrastare la carenza iodica [2]. In Italia, storicamente, la carenza iodica è stato un problema delle regioni montuose delle Alpi e degli Appennini. Studi successivi hanno tuttavia mostrato come la carenza iodica sia diffusa in tutta la penisola, in particolare nelle regioni del sud e insulari, riguardando fino al $65 \%$ della popolazione [3]. Uno studio di popolazione condotto a Pescopagano, piccolo borgo della Basilicata, ha mostrato come oltre il $50 \%$ della popolazione era affetto da gozzo e che la prevalenza aveva un andamento inverso con la iodio-carenza. Dopo quindici anni di iodio-profilassi, la prevalenza del gozzo nella stessa popolazione risultava tuttavia dimezzata [4]. Nel più recente report del 2020, l'Italia per la prima volta è un' area con adeguato apporto iodico [2, 5].

Da un punto di vista fisiopatologico, lo sviluppo del gozzo rappresenta un meccanismo compensatorio per garantire un'adeguata produzione di ormoni tiroidei. In uno schema molto semplificato, lo scarso apporto iodico determina un deficit nella produzione degli ormoni tiroidei e un conseguente incremento del TSH. Il continuo stimolo del TSH favorirebbe l'iperplasia e l'ipertrofia dei tireociti con accrescimento della ghiandola tiroidea e sviluppo del gozzo [1].

Numerosi studi hanno valutato quale sia il corretto apporto iodico in relazione all'età. Sulla base delle indicazioni del World Health Organization/International Council for the Control of Iodine Deficiency Disorders (WHO/ICCIDD), l' apporto iodico raccomandato aumenta con l'età ed è massimo in corso di gravidanza ed allattamento (Tabella 1) $[1,6]$.

Infine, è bene ricordare che il gozzo endemico è solo uno dei disordini correlati alla carenza iodica. Il deficit di iodio durante lo sviluppo fetale e nei primi anni di vita è responsabile di un alterato sviluppo del sistema nervoso centrale con quadri clinici che vanno dal ritardo mentale lieve a quello grave del cretinismo endemico, sebbene oggi queste condizioni siano assai rare [1].

\section{Gozzo disormogenico o familiare}

La disormogenesi tiroidea è l'incapacità della tiroide di produrre adeguate quantità di ormoni per la presenza di difetti genetici nelle proteine e negli enzimi che rivestono un ruolo chiave in questo processo. Circa il $15-20 \%$ dei casi di ipotiroidismo congenito sono ascrivibili alla disormogenesi tiroidea $[1,7]$. Il deficit di ormoni tiroidei determina l'incremento dei livelli di TSH e il conseguente sviluppo di gozzo che può essere presente già alla nascita o svilupparsi nei mesi successivi. Nella maggior parte dei casi la disormogenesi tiroidea è dovuta a difetti genetici a trasmissione autosomica recessiva e, essendo quasi sempre associata a ipotiroidismo, viene generalmente identificata alla nascita grazie al test di screening per l'ipotiroidismo congenito e trattata con levotiroxina [1]. Nelle forme più lievi lo screening alla nascita può tuttavia fallire con comparsa dell'ipotiroidismo e del gozzo più tardivamente, in quelle fasi della vita in cui è richiesto un fabbisogno maggiore di ormoni tiroidei.

I più comuni difetti genetici riguardano le proteine coinvolte nell'organificazione dello iodio (sodium-iodine symporter, NIS, pendrina, tireoperossidasi, TPO, dual oxidase 2, DUOX-2 e dual oxidase maturation factor 2, DUOXA2), nella sintesi della tireoglobulina ( $\mathrm{Tg}$ ) o nel "riciclo" intratiroideo dello iodio (iodio-tirosino deiodinasi 1, DEHAL1) [7, 8]. Altri difetti congeniti che possono provocare lo sviluppo di gozzo sono le mutazioni con acquisto di funzione del recettore del TSH (TSHR) che provocano l'iperplasia tiroidea tossica nelle due formi autosomica dominante o sporadica. Anche la resistenza agli ormoni tiroidei può rappresentare una causa genetica di gozzo diagnosticato però quasi sempre in età adulta [1].

È importante sottolineare come alcune forme di gozzo disormogenico si sviluppino nel contesto di quadri sindromici più complessi. Mutazioni a carico della pendrina, un trasportatore ionico espresso a livello tiroideo, renale e dell'orecchio interno, possono indurre lo sviluppo della Sindrome di Pendred, una patologia autosomica recessiva caratterizzata da sordità, gozzo e talora grave ipotiroidismo [8].

\section{Gozzo sporadico}

Si definisce "sporadico" il gozzo che insorge in aree iodiosufficienti. Il gozzo sporadico rappresenta quindi la forma più diffusa nei paesi sviluppati e nella maggior parte dei casi la sua eziologia è sconosciuta [1]. È verosimile che l'interazione di fattori ambientali e genetici concorrano al suo sviluppo [9]. Studi condotti in aree iodio-sufficienti hanno dimostrato che i gemelli monozigoti hanno una maggiore concordanza nello sviluppo di gozzo rispetto ai gemelli eterozigoti. Studi di linkage hanno poi permesso di identificare diversi loci associati a un maggior rischio di sviluppare il gozzo. Tra gli altri ricordiamo il locus del gozzo multinodulare $1(M N G-1)$ situato sul cromosoma $14 \mathrm{q} 31$ identificato in una famiglia canadese e il locus del gozzo multinodulare 2 $(M N G-2)$ identificato sul cromosoma Xp22 [9, 10]. 
Tabella 2 Principali sostanze naturali con noto effetto gozzigeno

\begin{tabular}{lll}
\hline Alimenti & Sostanza & Meccanismo di azione \\
\hline $\begin{array}{l}\text { Miglio } \\
\text { Soia }\end{array}$ & Flavonoidi & Blocco della tireoperossidasi \\
$\begin{array}{l}\text { Manioca } \\
\text { Patate dolci }\end{array}$ & Tiocianati & Inibizione dell'uptake dello iodio \\
$\begin{array}{l}\text { Saggina } \\
\text { Crucifere }\end{array}$ & \\
Alghe marine & Glucosinolati & Interferenza sull' uptake dello iodio \\
\hline
\end{tabular}

Oltre alla carenza iodica, altri fattori esogeni possono contribuire allo sviluppo del gozzo. Alcune sostanze "gozzigene" che inibiscono la sintesi degli ormoni tiroidei sono presenti in numerosi alimenti (Tabella 2). I semi delle Brassicaceae sono ricchi di queste sostanze e il loro ruolo nello sviluppo del gozzo in diverse aree dell' Africa è stato ben documentato (Tabella 2). I glicosidi cianogenetici contenuti nella manioca, nei broccoli, nelle rape, nel cavolo e nella colza vengono metabolizzati in prodotti che interferiscono con la captazione di iodio da parte della tiroide. La manioca, in particolare, se consumata non adeguatamente cotta, rilascia tiocianati che riducono l'ingresso di iodio nella tiroide (Tabella 2). Il fumo di sigaretta contiene elevate quantità di tiocianati ed è stato anche ipotizzato che l'apporto di iodio nel neonato possa essere compromesso dalla presenza di queste sostanze nel latte materno di madri fumatrici. I flavonoidi, riccamente contenuti nella soia e nei suoi derivati, interferiscono con l'attività della TPO. Più recentemente numerose sostanze contenute negli inquinanti ambientali sono state classificate come endocrine disruptors, come ad esempio i nitrati, il perclorato e il resorcinolo [11]. Anche l'eccesso di iodio può favorire lo sviluppo di gozzo in soggetti predisposti che esprimono polimorfismi genici in grado di ridurre la capacità di escape al blocco dell'organificazione dello iodio (effetto Wolff-Chaikoff), come è stato descritto in alcune aree del Giappone (Tabella 2). Infine, diversi studi hanno mostrato come il deficit di oligoelementi e vitamine come il selenio, il ferro e la vitamina A, possa peggiorare gli effetti della carenza iodica, aumentando il rischio di sviluppare il gozzo [1, 12].

\section{Patogenesi e storia naturale}

La differente incidenza di gozzo in aree con simile apporto iodico, il fatto che solo una parte di soggetti sviluppano il gozzo nelle aree iodio-carenti e lo sviluppo di gozzo anche in zone con adeguato o elevato apporto iodico fanno capire come nella patogenesi di questa malattia concorrano in modo variabile fattori endogeni ed esogeni. È stato dimostrato chiaramente che la carenza iodica rappresenta il fattore di rischio principale sia in esperimenti in vitro che in modelli animali. Sebbene sia stato osservato che lo iodio di per sé sia un inibitore della crescita dei tireociti in vitro, il principale meccanismo fisiopatologico vede nella carenza iodica il primum movens dell'alterata sintesi degli ormoni tiroidei e del conseguente incremento dei valori del TSH. Sotto lo stimolo del TSH il tireocita iperesprime il NIS sulla propria superficie aumentando così l'uptake di iodio e ristabilendo una sufficiente produzione di ormoni tiroidei [1, 12]. Nel caso in cui la carenza iodica sia protratta, questo meccanismo adattivo non riesce a compensare il deficit di ormoni tiroidei e i tireociti vanno incontro a proliferazione con conseguente sviluppo di gozzo. Lo sviluppo del gozzo sarebbe quindi un meccanismo di mancato adattamento, come dimostrato in numerosi modelli animali [13]. Il TSH è, infatti, il principale fattore di crescita per le cellule tiroidee e agisce principalmente attraverso vie di trasduzione del segnale mediate dall' AMP ciclico [12, 13]. Le cellule follicolari differiscono, tuttavia, nella loro capacità replicativa e nella capacità di produrre ormoni tiroidei. Questo può essere spiegato dall'osservazione che oltre il $70 \%$ dei noduli tiroidei originano da una singola mutazione somatica. Lo sviluppo clonale dei follicoli con elevata capacità replicativa favorirà lo sviluppo di noduli non funzionanti (freddi) o di noduli iperfunzionanti (caldi), nei quali la sintesi degli ormoni tiroidei sarà indipendente dallo stimolo del TSH [12, 13].

Riguardo ai noduli iperfunzionanti, sono stati in parte compresi i meccanismi molecolari sottostanti lo sviluppo dell'autonomia funzionale. Mutazioni somatiche a carico del TSHR o della proteina Gs $\alpha$ associata al recettore inducono l'attivazione costitutiva della via di trasduzione del segnale, indipendentemente dal legame con il TSH. Queste mutazioni sono state descritte nel 10-80\% degli adenomi tossici, con una maggiore incidenza soprattutto nelle aree iodio-carenti. Non è stata tuttavia evidenziata una correlazione genotipo-fenotipo, in quanto è verosimile che anche altri fattori agiscano a valle del TSHR. Con il progressivo incremento dimensionale e il perpetuarsi dell'autonomia funzionale, si assiste inizialmente a un lieve incremento dei valori circolanti di FT4 e di FT3 con valori del TSH solo lievemente ridotti. Successivamente, è possibile assistere alla comparsa di un quadro di ipertiroidismo franco con elevati livelli di ormoni tiroidei e valori soppressi del TSH $[1,13]$. 
Nella storia naturale del gozzo multinodulare è assai comune assistere alla lenta progressione dall'eutiroidismo all'ipertiroidismo e l'apporto iodico gioca un ruolo cruciale in questa evoluzione. Diversi studi, compreso quello di Pescopagano, hanno evidenziato come la carenza iodica favorisca lo sviluppo di gozzo multinodulare tossico soprattutto nei soggetti anziani, mentre la iodio-sufficienza si associ a un maggior rischio di ipotiroidismo [4]. In linea con questi dati, diversi studi di popolazione hanno confermato che la più comune causa di ipertiroidismo nelle aree iodio carenti è rappresentato dal gozzo multinodulare tossico mentre nelle aree iodio-sufficienti dal Morbo di Basedow [1].

Nella patogenesi dei noduli freddi, invece, prevalgono quelle mutazioni che conferiscono un vantaggio proliferativo in assenza, tuttavia, di un incremento nella sintesi degli ormoni tiroidei. Studi molecolari hanno mostrato come, oltre alla via del TSHR, altre vie siano coinvolte nello sviluppo dei noduli non funzionanti. Fra tutte vanno ricordate quelle dell' insulin-like grow factor 1 (IGF-1), dell'epidermal growth factor (EGF) e del fibroblast growth factor (FGF). Nelle aree con sufficiente apporto iodico, i noduli non funzionanti sono nella maggior parte dei casi rappresentati da lesioni singole nel contesto di una ghiandola tiroidea normale. In questi casi, il nodulo assume spesso le caratteristiche adenomatose con un'origine monoclonale, un pattern microfollicolare e la presenza di una capsula. È importante ricordare che in una piccola percentuale di questi adenomi è possibile identificare mutazioni tipiche della carcinogenesi tiroidea (ad esempio a carico di BRAF e RAS) e, pertanto, queste lesioni possono essere considerate a tutti gli effetti come lesioni pre-neoplastiche [1].

\section{Gestione clinica}

I sintomi del gozzo sono legati alla crescita dei noduli e all'eventuale sviluppo di ipertiroidismo. Molte volte, tuttavia, non vi è una correlazione diretta tra la crescita della ghiandola e i sintomi compressivi o da tireotossicosi. La crescita della tiroide può avvenire sia verso l'esterno, nella regione del collo con importante disagio estetico, ma anche verso il basso, nel mediastino anteriore, e non è infrequente, soprattutto negli anziani, che un voluminoso gozzo venga scoperto incidentalmente nel corso di esami di imaging del torace. Generalmente i noduli tiroidei di dimensioni inferiori ai $2 \mathrm{~cm}$ sono asintomatici. I sintomi insorgono per lo più quando vi è compressione della trachea e dell'esofago, in particolar modo nei gozzi con estrinsecazione mediastinica. La dispnea, lo stridore inspiratorio e la tosse sono provocati dalla compressione della trachea e tendono a peggiorare in posizione supina o durante l'esercizio fisico. La compressione esofagea con associata disfagia è molto più rara. La compressione dei nervi laringei può determinare la comparsa di disfonia, sebbene questo sintomo sia più tipico dell'infiltrazione del nervo provocato da lesioni maligne e meriti sempre una particolare attenzione [1]. All'esame obiettivo è fondamentale eseguire un'attenta palpazione della regione del collo al fine di valutare la presenza di noduli, la loro estensione e le loro caratteristiche. I noduli benigni sono generalmente mobili, elastici e lisci, a differenza di quelli maligni che appaiono più spesso di consistenza aumentata, duri e fissi sui piani superficiali e profondi. Il segno di Pemberton, ovvero la comparsa di turgore giugulare e cianosi del volto quando le braccia vengono innalzate, è indicativo di una compressione importante a livello mediastinico [1].

Alcuni esami di laboratorio sono imprescindibili nell'inquadramento di un soggetto con gozzo multinodulare. Questi comprendono il dosaggio della funzione tiroidea (FT4, FT3 e TSH) e il dosaggio della calcitonina (CT). Il dosaggio degli ormoni tiroidei e del TSH permette di valutare la presenza o meno di autonomia funzionale, identificando i soggetti con gozzo tossico. La CT è il marcatore del tumore midollare della tiroide che origina dalle cellule parafollicolari tiroidee. Sebbene altre condizioni fisiologiche o patologiche possano associarsi a un incremento della $\mathrm{CT}$, il suo dosaggio rimane lo strumento più sensibile e specifico, superiore anche all'esame citologico con agoaspirato, per identificare precocemente i soggetti affetti da carcinoma midollare [14].

L'ecografia è il gold standard per la valutazione strumentale dei pazienti con gozzo. È una procedura semplice da eseguire, a basso costo e non invasiva. Fornisce informazioni preziose circa il volume complessivo della ghiandola tiroidea, il numero e la localizzazione dei noduli, la loro posizione e le loro dimensioni. Inoltre, numerosi studi hanno identificato alcune caratteristiche ecografiche sospette per malignità. Tra queste, le più importanti sono l'ipoecogenicità del nodulo rispetto al parenchima circostante, la presenza di microcalcificazioni, la presenza di margini irregolari. Sulla base delle caratteristiche ecografiche e delle dimensioni viene stabilita la classe di rischio ecografico del nodulo ponendo o meno l'indicazione a eseguire l'esame citologico su agoaspirato, come previsto dalle linee guida internazionali [15]. La scintigrafia tiroidea eseguita con ${ }^{131} \mathrm{I},{ }^{123} \mathrm{I}$ o con ${ }^{99 \mathrm{~m}} \mathrm{Tc}$ è uno strumento utile per caratterizzare l'attività funzionale dei noduli. Essa permette di distinguere tra i noduli freddi, con basso o assente uptake del tracciante, da quelli caldi caratterizzati da elevato uptake. In genere, è consigliato eseguirla in soggetti con gozzo nodulare e funzione tiroidea indicativa di una possibile autonomia funzionale (valori di TSH ridotti o ai limiti inferiori della norma). Il dato scintigrafico è importante anche ai fini dell'esame citologico su agoaspirato in quanto i noduli caldi, dato il bassissimo rischio di malignità, non vengono generalmente sottoposti a questa procedura [1]. Altri esami radiologici come la TC senza mezzo di contrasto o la RM del collo possono essere 
Tabella 3 Possibilità terapeutiche in pazienti con gozzo multinodulare

Terapia conservativa

Terapia semi-soppressiva il TSH con levotiroxina

Trattamento chirurgico

Terapia radiometabolica con ${ }^{131} \mathrm{I}$

Alcolizzazione dei noduli

Termoablazione (laser o a radiofrequenza)
- Gozzi di piccole o medie dimensioni con noduli a citologia benigna in assenza di segni e sintomi compressivi

- Con lo scopo di ridurre la crescita dei noduli o limitarne la comparsa di nuovi in giovani individui

- Noduli maligni

- Noduli a citologia indeterminata a elevato rischio (TIR 3B)

- Casi selezionati di pazienti con noduli a citologia indeterminata a basso rischio (TIR 3A)

- Noduli benigni che determinano importanti segni o sintomi compressivi

- Adenomi tossici o gozzi multinodulari tossici

- Gozzi multinodulari non tossici di grandi dimensioni in pazienti con controindicazione all' intervento chirurgico

- Noduli cistici o prevalentemente cistici

- Pazienti con sintomi compressivi o problematiche estetiche in alternativa alla chirurgia utili in casi selezionati, soprattutto quando si devono stabilire i rapporti anatomici di voluminosi gozzi a estrinsecazione mediastinica. L'elastografia è una tecnica ecografica che permette di valutare l'elasticità dei noduli tiroidei [16]. Diversi studi hanno dimostrato che i noduli benigni sono dotati di elevata elasticità rispetto a quelli maligni, tendenzialmente più rigidi [17]. Questa differenza trova il suo corrispettivo anatomopatologico nella maggiore fibrosi dei noduli maligni [16]. Questa tecnica, se eseguita da personale esperto, può quindi fornire utili informazioni soprattutto in quei noduli in cui il risultato dell'esame citologico su agoaspirato non è stato dirimente (vedi dopo) [16].

L'esame citologico su agoaspirato rappresenta la metodica più sensibile e specifica per stabilire la natura dei noduli tiroidei. Non deve essere eseguito in modo estensivo su tutti i noduli tiroidei ma in modo selettivo sulla base delle caratteristiche ecografiche e, eventualmente, scintigrafiche. $\grave{E}$ fondamentale che la lettura citologica sia eseguita da anatomopatologi esperti di citologia tiroidea. La classificazione italiana degli esami citologici identifica le seguenti classi di rischio:

- TIR 1: non diagnostico;

- TIR 2: citologia benigna;

- TIR 3A: citologia indeterminata a basso rischio;

- TIR 3B: citologia indeterminata ad alto rischio;

- TIR 4: citologia sospetta per malignità;

- TIR 5: citologia diagnostica per malignità.

Il 60-65\% dei noduli tiroidei freddi è benigno. La casistica indeterminata (TIR 3A e TIR 3B) include principalmente i noduli ad architettura microfollicolare (adenomi follicolari, carcinomi follicolari, varianti follicolari del carcinoma papillare) in cui il rischio di malignità oscilla tra il 10 e il $40 \%$. In questa categoria ricade circa il $20 \%$ dei noduli tiroidei. Infine, il 5-10\% dei noduli rientra nelle categorie ad alto rischio di malignità (TIR 4 e TIR 5) [1].

\section{Trattamento}

L'unico trattamento profilattico in grado di prevenire lo sviluppo del gozzo è l'assunzione di adeguate quantità di iodio nel corso di tutta la vita. L'utilizzo di sale iodato rappresenta lo strumento più semplice per assumere una quantità sufficiente di iodio [4, 5]. Un'integrazione è sempre raccomandabile durante la gravidanza e l'allattamento.

In presenza di gozzo, la scelta del trattamento deve essere individualizzata sul singolo paziente tenendo conto della natura dei noduli tiroidei, della loro attività funzionale, delle dimensioni, della presenza di sintomi compressivi e delle comorbilità [1].

I gozzi di piccole o medie dimensioni con noduli benigni e normale funzione tiroidea vengono trattati conservativamente, eseguendo un periodico monitoraggio ecografico e della funzione tiroidea (Tabella 3) [18]. I gozzi molto grandi con associati sintomi compressivi o con noduli maligni sono indirizzati all'intervento chirurgico. Il trattamento radiometabolico con ${ }^{131}$ I rappresenta, invece, un'opzione terapeutica molto valida in caso di adenomi tossici o di gozzi multinodulari tossici [19]. Lo iodio radioattivo si concentra, infatti, quasi esclusivamente nei noduli con autonomia funzionale, determinandone la distruzione con risoluzione dell'ipertiroidismo e notevole riduzione dimensionale. Il trattamento radiometabolico può rappresentare anche un'opzione terapeutica in presenza di voluminosi gozzi nodulari non tossici, in pazienti non candidabili all'intervento chirurgico (Tabella 3). Diversi studi hanno mostrato come in questi soggetti si possa ottenere una riduzione fino al $60 \%$ del volume tiroideo anche per volumi di partenza molto elevati (> 100-150 mL) [1, 17]. I principali effetti avversi sono rappresentati dalla comparsa di una tiroidite distruttiva post-radioiodio con dolore e fastidio nella regione del collo in circa il 5\% dei soggetti o la comparsa di ipertiroidismo Basedow-like in circa il 3-4\% dei soggetti. Tra le complicanze nel lungo periodo va ricordato il possibile sviluppo di 
ipotiroidismo. Sono invece contrastanti i dati riguardanti il rischio, comunque minimo, di sviluppare cancro tiroideo o in altri distretti corporei [20]. Negli ultimi anni sono state sviluppate tecniche mini-invasive di termoablazione dei noduli tiroidei (a luce laser o radiofrequenza) in grado di ridurre le dimensioni dei noduli, utili in soggetti con grandi noduli tiroidei benigni non candidabili all'intervento chirurgico (Tabella 3) [21].

Infine, un discorso a parte va riservato alla terapia semisoppressiva della secrezione del TSH con levotiroxina. Rappresenta l'unico trattamento medico del gozzo e consiste nella somministrazione di dosi sovra-fisiologiche di ormone tiroideo allo scopo di ridurre i valori del TSH nella parte inferiore del range di normalità o lievemente al di sotto, al fine di rallentare la crescita dei noduli tiroidei e prevenirne lo sviluppo di nuovi. Il razionale di questa terapia si basa sulla riduzione dello stimolo proliferativo rappresentato dal TSH. Sebbene in letteratura i dati riguardanti l'efficacia di questo trattamento siano contrastanti, in linea con le ultime linee sulla gestione del nodulo tiroideo e sulla base della nostra esperienza clinica, riteniamo utile consigliarla (Tabella 3) in soggetti giovani con gozzi di piccole e medie dimensioni dopo un'attenta valutazione dei possibili effetti avversi cardiovascolari e ossei [22].

Funding Note Open access funding provided by Università di Pisa within the CRUI-CARE Agreement.

Conflitto di interesse Gli autori Alessandro Brancatella, Nicola Viola, Daniele Sgrò, Debora Ricci, Paolo Vitti, Ferruccio Santini e Francesco Latrofa dichiarano di non avere conflitti di interesse. Ferruccio Santini e Francesco Latrofa fanno parte del comitato di redazione.

Consenso informato Lo studio presentato in questo articolo non ha richiesto sperimentazione umana.

Studi sugli animali Gli autori di questo articolo non hanno eseguito studi sugli animali.

Nota della casa editrice Springer Nature rimane neutrale in riguardo alle rivendicazioni giurisdizionali nelle mappe pubblicate e nelle affiliazioni istituzionali.

Open Access This article is licensed under a Creative Commons Attribution 4.0 International License, which permits use, sharing, adaptation, distribution and reproduction in any medium or format, as long as you give appropriate credit to the original author(s) and the source, provide a link to the Creative Commons licence, and indicate if changes were made. The images or other third party material in this article are included in the article's Creative Commons licence, unless indicated otherwise in a credit line to the material. If material is not included in the article's Creative Commons licence and your intended use is not permitted by statutory regulation or exceeds the permitted use, you will need to obtain permission directly from the copyright holder. To view a copy of this licence, visit http://creativecommons.org/licenses/by/4.0/.

\section{Bibliografia}

1. Latrofa F, Vitti P (2012) Diffuse and nodular goiter. In: Monaco F (ed) Thyroid diseases. Routledge Taylor \& Francis Group, Boca Raton, pp 9-47

2. The Iodine Global Network (2020) Global scorecard of iodine nutrition in 2020 in the general population based on school-age children (SAC). IGN, Ottawa

3. Aghini-Lombardi F, Vitti P, Antonangeli L et al (2013) The size of the community rather than its geographical location better defines the risk of iodine deficiency: results of an extensive survey in Southern Italy. J Endocrinol Invest 36(5):282-286

4. Aghini Lombardi F, Fiore E, Tonacchera M et al (2013) The effect of voluntary iodine prophylaxis in a small rural community: the Pescopagano survey 15 years later. J Clin Endocrinol Metab 98(3):1031-1039

5. Olivieri A, Trimarchi F, Vitti P (2020) Global iodine nutrition 2020: Italy is an iodine sufficient country. J Endocrinol Invest 43(11):1671-1672

6. International Council for Control of Iodine Deficiency Disorders (2008) UNICEF Report 2008. IDD Newsl 30

7. Spitzweg C, Morris JC (2010) Genetics and phenomics of hypothyroidism and goiter due to NIS mutations. Mol Cell Endocrinol 322(1-2):56-63

8. Bizhanova A, Kopp P (2010) Genetics and phenomics of Pendred syndrome. Mol Cell Endocrinol 322(1-2):83-90

9. Gozu HI, Lublinghoff J, Bircan R, Paschke R (2010) Genetics and phenomics of inherited and sporadic non-autoimmune hyperthyroidism. Mol Cell Endocrinol 322(1-2):125-134

10. Bayer Y, Neumann S, Meyer B et al (2001) Genome-wide linkage analysis reveals evidence for four new susceptibility loci for familial euthyroid goiter. J Clin Endocrinol Metab 89(8):4044-4052

11. Brix TH, Hegedüs L (2000) Genetic and environmental factors in the aetiology of simple goitre. Ann Med 32:153-156

12. Krohn K, Führer D, Bayer Y et al (2005) Molecular pathogenesis of euthyroid and toxic multinodular goiter. Endocr Rev 26(4):504-524

13. Studer H, Peter HJ, Gerber H (1989) Natural heterogeneity of thyroid cells: the basis for understanding thyroid function and nodular goiter growth. Endocr Rev 10(2):125-135

14. Elisei R (2008) Routine serum calcitonin measurement in the evaluation of thyroid nodules. Best Pract Res Clin Endocrinol Metab 22(6):941-953

15. Rago T, Cantisani V, Ianni F et al (2018) Thyroid ultrasonography reporting: consensus of Italian Thyroid Association (AIT), Italian Society of Endocrinology (SIE), Italian Society of Ultrasonography in Medicine and Biology (SIUMB) and Ultrasound Chapter of Italian Society of Medical Radiology (SIRM). J Endocrinol Invest 41(12):1435-1443

16. Rago T, Vitti P (2014) Diagnostic role of ultrasound and elastosonography in nodular goiter. Best Pract Res Clin Endocrinol Metab 28(4):519-529

17. Rago T, Scutari M, Loiacono V et al (2017) Low elasticity of thyroid nodules on ultrasound elastography is correlated with malignancy, degree of fibrosis, and high expression of galectin- 3 and fibronectin-1. Thyroid 27(1):103-110

18. Gharib H, Papini E, Garber JR et al (2016) American association of clinical endocrinologists, American college of endocrinology, and Associazione medici endocrinologi medical guidelines for clinical practice for the diagnosis and management of thyroid nodules - 2016 update. Endocr Pract 22(5):622-639

19. Roque C, Santos FS, Pilli T et al (2020) Long-term effects of radioiodine in toxic multinodular goiter: thyroid volume, function, and autoimmunity. J Clin Endocrinol Metab 105(7):dgaa214 
20. Kitahara CM, Berrington de Gonzalez A, Bouville A et al (2019) Association of radioactive iodine treatment with cancer mortality in patients with hyperthyroidism. JAMA Intern Med 179(8):1034-1042

21. Papini E, Rago T, Gambelunghe G et al (2014) Long-term efficacy of ultrasound-guided laser ablation for benign solid thyroid no- dules. Results of a three-year multicenter prospective randomized trial. J Clin Endocrinol Metab 99(10):3653-3659

22. Fiore E, Rago T, Provenzale MA et al (2010) L-thyroxine-treated patients with nodular goiter have lower serum TSH and lower frequency of papillary thyroid cancer: results of a cross-sectional study on 27914 patients. Endocr-Relat Cancer 17(1):231-239 